\title{
Two-photon photoluminescence and excitation spectra of InGaN/GaN quantum wells
}

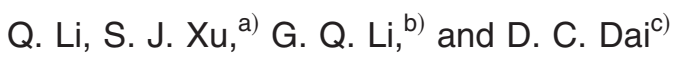 \\ Department of Physics, The University of Hong Kong, Pokfulam Road, Hong Kong, China \\ and HKU-CAS Joint Laboratory on New Materials, The University of Hong Kong, \\ Pokfulam Road, Hong Kong, China \\ C. M. Che \\ Department of Chemistry, The University of Hong Kong, Pokfulam Road, Hong Kong, China \\ and HKU-CAS Joint Laboratory on New Materials, The University of Hong Kong, \\ Pokfulam Road, Hong Kong, China
}

(Received 12 February 2006; accepted 27 May 2006; published online 6 July 2006)

\begin{abstract}
We report an observation of efficient two-photon photoluminescence (TPL) of InGaN/GaN multi-quantum-well (MQW) structures using broadband femtosecond near-infrared excitation laser. Near quadratic excitation-intensity dependence and asymmetric collinear interferometric autocorrelation trace of the TPL signal unambiguously verify the nonlinearity of the TPL process. We also measured the excitation spectrum of the TPL signal and found that it can be fitted well with the theoretical two-photon absorption coefficient formula for direct wide gap semiconductors. The decay time of the TPL signal was determined using a time-resolved photoluminescence technique. These results demonstrate the strong nonlinear optical property of InGaN/GaN MQWs. (C) 2006 American Institute of Physics. [DOI: 10.1063/1.2218772]
\end{abstract}

Two-photon absorption induced photoluminescence (TPL) is an important nonlinear optical phenomenon. It is not only of fundamental interest but also has many important applications such as two-photon microscopy, 1,2 threedimensional optical data storage, ${ }^{3}$ optical power limiting, ${ }^{4}$ single molecule detection, ${ }^{5}$ and fluorescence correlation spectroscopy in biological systems. ${ }^{6,7}$ Therefore, TPL phenomenon in various materials as well as their microstructures has been attracting significant attention since it was observed decades ago.

In the past decade, due to its central role as the active layers in $\mathrm{GaN}$ based short-wavelength light-emitting devices including laser diodes, ${ }^{8}$ the linear optical properties of InGaN/GaN multiquantum wells (MQWs) have been extensively investigated and well documented. ${ }^{9-12}$ Recently, nonlinear optical properties of $\mathrm{GaN}$ based materials and heterostructures have attracted an increasing interest. ${ }^{13-18}$ For example, Cingolani et al. measured two-photon absorption spectroscopy of GaN/AlGaN QWs (Ref. 14) and Satake et al. reported ultraviolet anti-Stokes photoluminescence in InGaN/GaN QW structures. ${ }^{17}$ On the other hand, available theoretical studies predicted that there exists a strong nonlinear optical effect in InGaN/GaN MQWs. ${ }^{16}$ Motivated by the wide applications of the TPL and the technological importance of InGaN/GaN layered structures, we experimentally investigated the TPL effect of InGaN/GaN MQWs at $77 \mathrm{~K}$. Observation of the intense TPL signal and its asymmetric interferograms unambiguously demonstrates the strong nonlinear optical effect of InGaN/GaN MQWs.

The sample used in the present study was grown on sapphire by metalorganic vapor phase epitaxy. A $30 \mathrm{~nm}$ lowtemperature $\mathrm{GaN}$ buffer was firstly grown, followed by $1 \mu \mathrm{m} \mathrm{GaN}$ epilayer. Ten periods of $3 \mathrm{~nm} \mathrm{In}_{0.13} \mathrm{Ga}_{0.87} \mathrm{~N} / 5 \mathrm{~nm}$

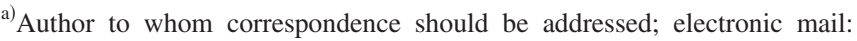
sjxu@hkucc.hku.hk

${ }^{b}$ Present address: Technische Universität Chemnitz, Germany.

${ }^{c)}$ Present address: Durham University, UK.
}

GaN MQWs were then grown, which was capped by a $20 \mathrm{~nm}$ GaN layer. Near infrared femtosecond laser pulses with $80-100$ pulse width from a Ti:sapphire oscillator (Tsunami, Spectra-Physics) pumped by solid-state laser diode array were employed to excite the sample at $77 \mathrm{~K}$. The wavelengths of the laser pulses can be tuned from 710 to $950 \mathrm{~nm}$. The luminescence signal from the sample was dispersed with a grating spectrometer (Acton SP300) and detected with photomultiplier (R928, Hamamatsu). A digital phase lock-in amplifier (SR850, Stanford Research) was used to enhance the signal-to-noise ratio. Time-resolved PL spectra were measured with a $25 \mathrm{~cm}$ spectrometer and a synchroscan streak camera (C4334, Hamamatsu). The collinear interferometric autocorrelation traces of the luminescence signal were recorded using an experimental arrangement based on Michelson interferometer. ${ }^{19}$

Figure 1 shows representative three $77 \mathrm{~K}$ TPL spectra from the sample excited by $710 \mathrm{~nm}(\sim 1.746 \mathrm{eV}), 760 \mathrm{~nm}$ $(\sim 1.632 \mathrm{eV})$, and $780 \mathrm{~nm}(\sim 1.590 \mathrm{eV})$ femtosecond pulses. The TPL spectra are dominated by a luminescence peak at $425 \mathrm{~nm}(\sim 2.918 \mathrm{eV})$. The inset logarithmically shows the integrated TPL intensity versus the excitation power of $760 \mathrm{~nm}$ laser. The power index obtained from the leastsquares fitting using a power function is $2.12 \pm 0.07$, which is slightly more than the ideal value of 2 . It is well known that the integrated TPL intensity depends on the excitation intensity in a quadratic law. ${ }^{20}$ However, recent studies about the excitation intensity dependence of the TPL in the organic chromophores in solution show that validity of the quadratic law is questioned for the cases of strong excitation. ${ }^{21} \mathrm{We}$ thus carefully seek for more and clearer evidences to verify the nonlinearity of the TPL in the InGaN/GaN MQWs. For the purpose, we measured the collinear interferometric autocorrelation traces of the TPL signal. In an earlier work, such method has been employed to investigate the multiphoton absorption induced luminescence in $\mathrm{ZnO}$ single crystal. ${ }^{22}$ 


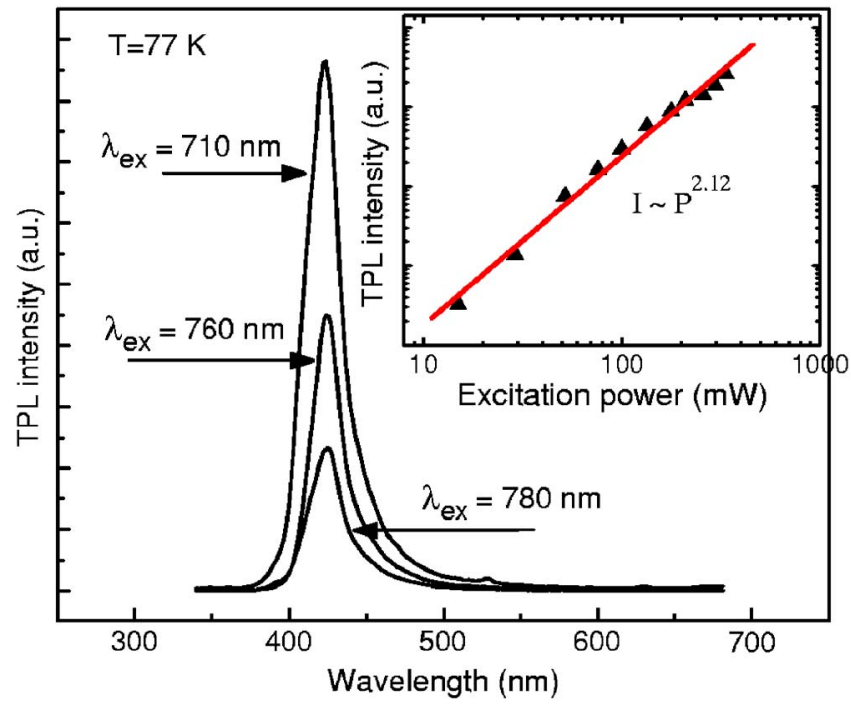

FIG. 1. (Color online) Representative three TPL spectra of the sample at $77 \mathrm{~K}$. The average powers of excitation pulses with different wavelengths are kept as $283 \mathrm{~mW}$. The inset shows the logarithmic excitation-power dependence of the integrated TPL intensity for excitation wavelength of $760 \mathrm{~nm}$. The power index obtained from the least-squares fitting is $2.12 \pm 0.07$.

A representative collinear interferometric autocorrelation trace of the TPL signal recorded for the sample at $77 \mathrm{~K}$ was shown in Fig. 2(a), whereas Fig. 2(b) depicts a theoretical second-order autocorrelation trace of two Gaussian excitation pulses with varying time delay propagating along the same direction in medium. Clearly, the measured interferogram exhibits a strong asymmetry in intensity, which is consistent with the theoretical curve. The minimum TPL signal approaches zero while the maximum luminescence signal at zero delay time is $\sim 6.6$ times stronger than that at long enough delay time. Despite the fact that the intensity ratio between the maximum TPL signal at zero delay time and that at long enough delay time is not exactly equal to the theoretical value of 8 for the ideal second-order nonlinear process, ${ }^{23}$ the result still evidently demonstrates that the observed TPL signal really originates from the efficient twophoton absorption. The deviation of measured intensity ratio to the theoretical value may be due to the involvement of defect states in the two photon absorption process. ${ }^{22} \mathrm{Al}-$ though the traces shown in Figs. 2(a) and 2(b) are very similar to each other, a more careful comparison between the subfemtosecond interference fringes of the two traces shows

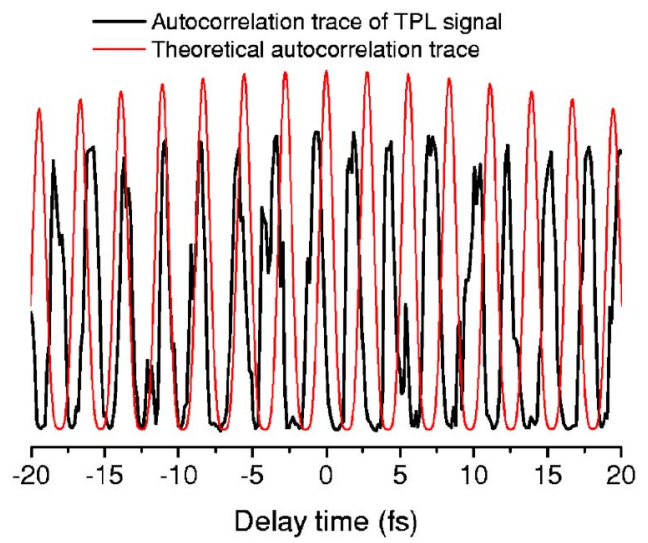

FIG. 3. (Color online) Closer look of measured TPL interferometric autocorrelation trace (thicker line) and theoretical second-order autocorrelation trace (thiner line) of two Gaussian excitation pulses.

that phase shift of the luminescence signal with respect to the excitation pulse takes place, as shown in Fig. 3. The experiment of Yu et al. has demonstrated the existence of the phase shift of the free-induction decay relative to the excitation pulses in GaAs. ${ }^{24}$ Detailed discussion about the phase shift of TPL signal is beyond the scope of the present short letter.

It is known that TPL intensity is proportional to twophoton absorption coefficient in semiconductors. ${ }^{25}$ In order to further investigate the properties of the TPL process in the InGaN/GaN MQWs, the excitation spectrum (open triangles) of the TPL signal at $425 \mathrm{~nm}$ was measured at fixed excitation power, as shown in Fig. 4. As expected, the TPL intensity rapidly increases with increasing energy of exciton photons. For direct band gap semiconductors, theoretical absorption coefficient is well established as ${ }^{26,27}$

$$
\beta^{(2)}\left(\omega_{p}\right)=K_{p b} \frac{\sqrt{E_{p}}}{n^{2}\left(\omega_{p}\right) E_{g}^{3}} \frac{(2 x-1)^{3 / 2}}{(2 x)^{5}},
$$

where $K_{p b}=3100 \mathrm{~cm} / \mathrm{GW}(\mathrm{eV})^{5 / 2}$ is the material independent parameter, ${ }^{27} E_{p}$ is related to the interband momentum matrix element and approximately $21 \mathrm{eV}$ for most semiconductors, ${ }^{27} n\left(\omega_{p}\right)$ is the refractive index of material at $\omega_{p}$, and $x=\hbar \omega_{p} / E_{g}$. Note that Eq. (1) is valid only for $x$ $\geqslant \frac{1}{2}$. Using Eq. (1), we did fitting to the experimental data and the fitting curve was depicted as solid line in Fig. 4. Reasonably good agreement between the experimental data and the theoretical fitting curve is achieved for $E_{g}=3.01 \mathrm{eV}$
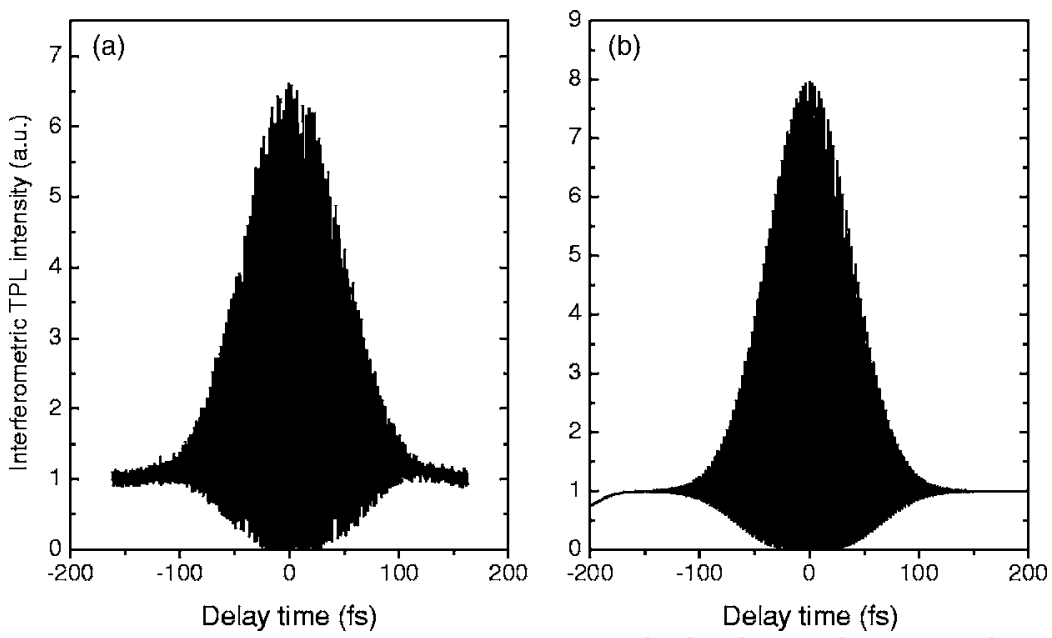

FIG. 2. (a) Representative collinear interferometric autocorrelation trace of the TPL signal from the sample at $77 \mathrm{~K}$ using the experimental arrangement based on Michelson interferometer; (b). Theoretical second-order autocorrelation trace of two Gaussian excitation pulses propagating along the same direction in medium. 


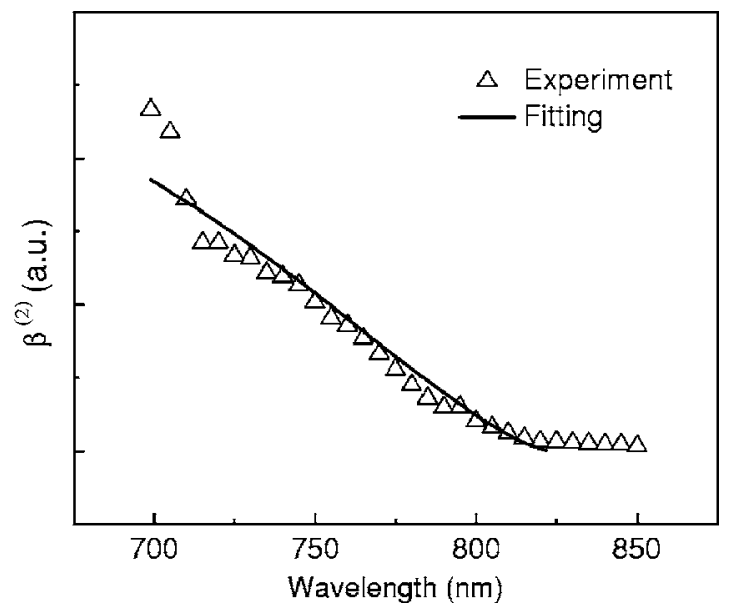

FIG. 4. Excitation spectrum (open triangles) of the TPL peak at $425 \mathrm{~nm}$ from the sample at $77 \mathrm{~K}$. The solid line is a least-squares fitting curve using theoretical two-photon absorption coefficient formula described in the text.

(corresponding wavelength of $\sim 412 \mathrm{~nm}$ ). Again, this proves that the two-photon absorption is responsible for the efficient TPL signal in the sample. It is worth mentioning that in the view of microscopic point, two-photon absorption coefficients of materials are related with the third-order nonlinear susceptibility tensor $\chi^{(3)}$ of materials. The two-photon absorption is thus usually regarded as the third-order nonlinear optical process ${ }^{28}$ although the intensity of two-photon absorption depends quadratically upon the intensity of incident excitation light.

At last, we would like to know the lifetime of photogenerated carriers due to the two-photon absorption. To this end, we measured time-resolved TPL spectra using the standard streak-camera technique. It was found that the TPL intensity exponentially decays as a function of time after the excitation of the $760 \mathrm{~nm}$ laser pulses. A time constant of $\tau$ $=0.69 \mathrm{~ns}$ is found for the TPL peak. For comparison, the decay curve of the normal PL signal from the sample under excitation of $355 \mathrm{~nm}$ laser pulses which are obtained by doubling frequency of the fundamental $710 \mathrm{~nm}$ femtosecond laser using a BBO (beta barium borate) crystal was also measured. In contrast to the TPL signal, the normal PL intensity exhibits a biexponential decay with time. By doing the biexponential fitting to the normal PL decaying curve, two time constants of $\tau_{1}=0.272 \mathrm{~ns}$ and $\tau_{2}=0.90 \mathrm{~ns}$ are yielded. In general, the bi- or multiexponential decay implies that the multilevels could be involved in the radiative recombination of carriers. ${ }^{29}$ Under the excitation of $355 \mathrm{~nm}$ laser pulses, the strong single-photon absorption takes place in the GaN cap and barrier layers in the sample as the single photon energies are already higher than the fundamental gap of GaN. It has been documented that a significant portion of the photogenerated carriers would be transfered from the GaN layers to the InGaN well layers. ${ }^{30}$ Such carrier transfer and the broad energy distribution of the localized states involved in the radiative recombination should be responsible for the observed biexponential decay of the normal PL of the sample. Under the conditions in which the two-photon absorption occurs only in the InGaN well layers, the carriers shall not experience the transfer process and the single exponential decay of the TPL signal is thus expected.

In summary, efficient two-photon absorption induced luminescence was observed in In-GaN/GaN MQWs at $77 \mathrm{~K}$. Measurements of excitation-intensity dependence, collinear interferometric autocorrelation trace, and excitation spectrum of the TPL signal unambiguously demonstrate that the strong two-photon absorption is responsible for the efficient TPL signal. These results show that $\mathrm{InGaN} / \mathrm{GaN}$ MQWs structures are promising heterostructures for applications of nonlinear optics.

The work was supported by Hong Kong Research Grants Council-Competitive Earmarked Research Grants under Contract No. HKU-7036/03P and the Research Grants of the University of Hong Kong (HKU) under Contract No. 10205780, as well as partially by the University Development Funding of HKU under Contract No. 00600009. One of the authors (S.J.X.) wishes to acknowledge Professor S. J. Chua's group for providing the samples studied in the present work.

${ }^{1}$ W. Denk, J. H. Strickler, and W. W. Webb, Science 248, 73 (1990).

${ }^{2}$ S. P. Tai, M. C. Chan, T. H. Tsai, S. H. Guol, L. J. Chen, and C. K. Sun, Opt. Express 12, 6122 (2004).

${ }^{3}$ J. H. Strickler and W. W. Webb, Opt. Lett. 16, 1780 (1991).

${ }^{4}$ J. E. Ehrlich, X. L. Wu, I.-Y. S. Lee, Z.-Y. Hu, H. Rockelm, S. R. Marder, and J. W. Perry, Opt. Lett. 22, 1843 (1997).

${ }^{5}$ J. Mertz, C. Xu, and W. W. Webb, Opt. Lett. 20, 2532 (1995).

${ }^{6}$ K. M. Berland, P. T. C. So, and E. Gratton, Biophys. J. 68, 694 (1995).

${ }^{7}$ P. Schwille, U. Haupts, S. Maiti, and W. W. Webb, Biophys. J. 77, 2251 (1999).

${ }^{8}$ S. Nakamura, S. Pearton, and G. Fasol, The Blue Laser Diode (Springer, Berlin, 2000).

${ }^{9}$ Introduction to Nitride Semiconductor Blue Lasers and Light Emitting Diodes, edited by S. Nakamura and S. F. Chichibu (Taylor \& Francis, London, 2000)

${ }^{10}$ III-Nitride Semiconductors: Optical Properties, edited by M. O. Manasreh and H. X. Jiang (Taylor \& Francis, New York, 2002).

${ }^{11}$ H. Morkoç, Nitride Semiconductors and Devices (Springer, Berlin, 1999).

${ }^{12}$ Nitride Semiconductors: Handbook on Materials and Devices, edited by P. Ruterana, M. Albrecht, and J. Neugebauer (Wiley-VCH, Weinheim, 2002).

${ }^{13}$ S. J. Sheih, K. T. Tsen, D. K. Ferry, A. Botchkarev, B. Sverdlov, A. Salvador, and H. Morkoc, Appl. Phys. Lett. 67, 1757 (1995).

${ }^{14}$ R. Cingolani, G. Coli, R. Rinaldi, L. Calcagnile, H. Tang, A. Botchkarev, W. Kim, A. Salvador, and H. Morkoç, Physica E (Amsterdam) 2, 539 (1998).

${ }^{15}$ C. K. Sun, J. C. Liang, J. C. Wang, F. J. Kao, S. Keller, M. P. Mack, U. Mishra, and S. P. DenBaars, Appl. Phys. Lett. 76, 439 (2000).

${ }^{16}$ S. Krishnamurthy, K. Nashold, and A. Sher, Appl. Phys. Lett. 77, 355 (2000).

${ }^{17}$ A. Satake, Y. Masumoto, T. Miyajima, T. Asatsuma, and T. Hino, Phys. Rev. B 61, 12654 (2000).

${ }^{18}$ S. W. Chu, M. C. Chan, S. P. Tai, S. Keller, S. P. DenBaars, and C. K. Sun, Opt. Lett. 30, 2463 (2005).

${ }^{19}$ Q. Li, S. J. Xu, D. C. Dai, and C. M. Che, IEEE Proceedings of the COMMAD04 (IEEE, Piscataway, NJ, 2005), p. 153.

${ }^{20}$ See, for example, C. Xu and W. W. Webb, J. Opt. Soc. Am. B 13, 481 (1996).

${ }^{21}$ C. H. Wang, O. Y.-H. Tai, Y. Wang, T.-H. Tsai, and N.-C. Chang, J. Chem. Phys. 122, 084509 (2005).

${ }^{22}$ D. C. Dai, S. J. Xu, S. L. Shi, M. H. Xie, and C. M. Che, Opt. Lett. 30, 3377 (2005).

${ }^{23}$ See, for example, Femtosecond Laser Pulses: Principles and Experiments, edited by C. Rulliere (Springer, Berlin, 1998).

${ }^{24}$ X. Y. Yu, Q. Luo, W. L. Li, Q. Li, Z. R. Qiu, and J. Y. Zhou, Appl. Phys. Lett. 73, 3321 (1998).

${ }^{25}$ M. Lepore, A. Adinolfi, M. C. Netti, I. Catalano, and I. Suemunez, J. Phys.: Condens. Matter 9, 7667 (1997).

${ }^{26}$ B. S. Wherrett, J. Opt. Soc. Am. B 1, 67 (1984).

${ }^{27}$ D. C. Hutchings and E. W. Van Stryland, J. Opt. Soc. Am. B 9, 2065 (1992).

${ }^{28}$ J. D. Bhawalkar, G. S. He, and P. N. Prasad, Rep. Prog. Phys. 59, 1041 (1996).

${ }^{29}$ S. J. Xu, M. B. Yu, Rusli, S. F. Yoon, and C. M. Che, Appl. Phys. Lett. 76, 2550 (2000).

${ }^{30}$ E. S. Jeon, V. Kozlov, Y.-K. Song, A. Vertikov, M. Kuball, A. V. Nurmikko, H. Liu, C. Chen, R. S. Kern, C. P. Kuo, and M. G. Craford, Appl. Phys. Lett. 69, 4194 (1996).

to AIP license or copyright, see http://apl.aip.org/apl/copyright.jsp 